RESEARCH METHODS

\title{
A Comparison of AGREE and RIGHT: which Clinical Practice Guideline Reporting Checklist Should Be Followed by Guideline Developers?
}

\author{
Xiaomei Yao, MD, MSc ${ }^{1,2,3}$, Jinhui Ma, $P h D^{2}$, Qi Wang, MMed, $P h D(c)^{2}$, \\ David Kanters, $M S c^{2}$, Muhammad U. Ali, MD, MSc, $P h D(c)^{2}$, and Ivan D. Florez, MD, MSc, \\ $\operatorname{PhD}(c)^{2,4,5}$ (D)
}

'Department of Oncology, McMaster University, Hamilton, Ontario, Canada; ${ }^{2}$ Department of Health Research Methods, Evidence, and Impact, McMaster University, Hamilton, Ontario, Canada; ${ }^{3}$ Clinical Practice Guideline Conduction and Evaluation Centre, Children's Hospital of Fudan University, Shanghai, China; ${ }^{4}$ Department of Pediatrics, University of Antioquia, Medellín, Colombia; ${ }^{5}$ Department of Pediatrics, School of Medicine, University of Antioquia, Medellín, Colombia.

BACKGROUND: A clinical practice guideline (CPG) reporting checklist is used to assist CPG developers in recording what content should be provided in a CPG report. Recently, two checklists have become available on the Enhancing the QUAlity and Transparency Of health Research Network website: AGREE (Appraisal of Guidelines, Research and Evaluation) published in 2016 and RIGHT (Reporting Items for practice Guidelines in HealThcare) published in 2017. The objective of this study was to describe the advantages and disadvantages of these two CPG reporting checklists.

METHODS: Two epidemiologists who lacked experience using both AGREE and RIGHT but were familiar with evidence-based medicine methodology independently compared AGREE with RIGHT on an item-by-item basis. Their assessments were compiled on a pre-designed data form and any disagreements were resolved through discussion. Three other co-authors independently compared AGREE with RIGHT and decided if they agreed with the results of comparison of the two CPG reporting checklists from the first two co-authors. Finally, another co-author reviewed the comparison results to ensure that the description was clear and understandable.

RESULTS: The following six relationships between the two checklists were observed: (1) 11 items from AGREE completely matched with 12 items from RIGHT; (2) four items were listed in AGREE only; (3) 12 items were listed in RIGHT only; (4) three items in AGREE were partially covered by three items in RIGHT; (5) six items in RIGHT were partially covered by three items in AGREE; and (6) two items intersected across AGREE and RIGHT. Based on the comparison results, the potential impact analysis of selecting either checklist is described.

DISCUSSION: We recommend that CPG developers use either AGREE plus items unique to RIGHT or RIGHT plus items unique to AGREE.

KEY WORDS: clinical practice guideline reporting checklist; AGREE reporting checklist; RIGHT reporting checklist; EQUATOR Network.

Received October 8, 2019

Revised October 8, 2019

Accepted October 16, 2019

Published online November 11, 2019
$\mathrm{J}$ Gen Intern Med 35(3):894-8

DOI: $10.1007 / \mathrm{s} 11606-019-05508-3$

(c) Society of General Internal Medicine 2019

\section{INTRODUCTION}

Clinical practice guidelines (CPGs) are systematically developed statements that aim to assist stakeholders in making appropriate decisions regarding healthcare for specific clinical circumstances ${ }^{1}$. High-quality CPGs are essential in bridging gaps between policy, best practice, and patient choice, to enhance healthcare quality and patient outcomes ${ }^{1}$. From a methodological perspective, three types of methodological tools/methods help CPG developers and authors to conduct high-quality CPGs: how to assess the quality of existing relevant CPGs with your topic, how to conduct a CPG, and how to report a CPG after completing it.

The AGREE (Appraisal of Guidelines, REsearch and Evaluation) II assessment tool is a widely used tool used to appraise the quality of existing CPGs. AGREE II assessment tool was updated from its original 2003 version and published in $2010^{2}$. The GRADE (Grading of Recommendations, Assessment, Development and Evaluations) approach is a widely accepted methodology to present the quality of evidence (also called "certainty" of evidence) and generate recommendations in the context of CPG development ${ }^{3}$. A guideline reporting checklist is intended to aid guideline authors (including methodologists and clinicians) and journal editors with the final guideline reporting manuscript once the CPG is finished, which is different from a conduction handbook (GRADE approach) or an assessment tool (AGREE II). How to clearly report the process with key steps after completing a CPG and to maintain transparency is important because $\mathrm{CPG}$ users will judge whether it is a high-quality CPG based on the reporting details, which will impact CPG users' confidence on implementing the CPG in the clinical practice.

The EQUATOR (Enhancing the QUAlity and Transparency Of health Research) Network (http://www.equator-network.org/) 
is an international initiative promoting transparent and accurate reporting statements for original studies, systematic reviews, and CPGs, to improve the quality and reliability of health research literature ${ }^{4}$. The EQUATOR Network is the only recommended resource by the International Committee of Medical Journal Editors (ICMJE) to guide authors and medical journal editors on which reporting checklist should be used when preparing a scientific manuscript. Currently, two reporting checklists are available at the EQUATOR Network website for CPGs: the AGREE checklist, published in $2016^{5}$, and the RIGHT (Reporting Items for practice Guidelines in HealThcare) checklist, published in $2017^{6}$. The AGREE reporting checklist ${ }^{5}$ was derived from the AGREE II CPG assessment tool ${ }^{2}$, but they remain as two independent tools used for different purposes by the end users. The authors of RIGHT reporting checklist intended to add new items and provide detailed information and examples that AGREE reporting tool lacks ${ }^{6}$. CPG developers and authors often struggle with selecting between them because they are unsure about the advantages and disadvantages of each checklist. The objective of this study was to illustrate the differences between the two CPG reporting checklists of AGREE and RIGHT and to discuss their potential impact on the reporting quality of CPGs.

\section{METHODS}

Two epidemiologists (DK and AU) who lacked experience using either AGREE or RIGHT but were familiar with Evidence-Based Medicine methodology and fluent in English, independently compared AGREE with RIGHT on an item-by-item basis to determine if and how these two reporting checklists differed. Their assessments were compiled on a pre-designed data form and any disagreements were resolved through discussion. Three other co-authors (XY and IF, familiar with AGREE; QW, familiar with RIGHT) independently compared AGREE with RIGHT again and decided if they agreed with the results of comparison of these two CPG reporting checklists from the first two coauthors. Then, five co-authors reached a consensus by discussion. Finally, another co-author (JM), a methodologist and statistician without experience of conduction a CPG and using either CPG reporting checklist, reviewed the comparison results of AGREE and RIGHT to ensure that the description was clear and understandable.

\section{RESULTS}

The AGREE and RIGHT reporting checklists present similar content using different structures. AGREE has 23 items with 2-7 reporting criteria to evaluate under each item ${ }^{5}$. The RIGHT checklist has 22 topics with 1-3 items under each topic (a total of 35 items) ${ }^{6}$. The following six relationships between the two checklists were observed: (1) 11 items (items
$1,2,5,9,10,11,15,16,17,20$, and 23) from AGREE completely matched with 12 items from RIGHT (items 6, $8 \mathrm{~b}, 10 \mathrm{a}, 14 \mathrm{a}, 12,15,13 \mathrm{a}, 13 \mathrm{~b}, 2,14 \mathrm{~b}, 19 \mathrm{a}$, and 19b) (Table 1); (2) four items (items 8, 14, 18, and 21) were listed in AGREE only; (3) 12 items (items 1a, 1b, 1c, 3, 4, 5, 10b, 11a, 14c, 17, 21, and 22) were listed in RIGHT only; (4) three items (items 6, 7, and 13) in AGREE were partially covered by three items in RIGHT (items 8a, 11b, and 16); (5) six items (items 7a, 7b, 9a, 9b, 18a, and 18b) in RIGHT were partially covered by three items (items 3, 4, and 22) in AGREE; and (6) two items intersected across AGREE (items 12 and 19) and RIGHT (items 13c and 20) (Table 2). Based on the comparison results, the potential impact analysis of selecting either checklist is indicated in Table 2.

\section{DISCUSSION}

Both AGREE and RIGHT checklists have their own strengths and limitations. Approximately half of the items from the AGREE and RIGHT checklists completely overlap. The unique items of RIGHT emphasize using presentation format, i.e., elements to include in a CPG title, a list of abbreviations and acronyms, and contact information of the corresponding developer; these items are good reminders for CPG developers. "How the outcomes were selected and sorted" increases CPG clarity for how to choose outcomes and rank them among each other.

Table 1 Completely Matched Items Between AGREE and RIGHT

\begin{tabular}{|c|c|}
\hline AGREE (item)* & RIGHT (item) $\dagger$ \\
\hline 1. Objectives & $\begin{array}{l}\text { 6. Aims of the guideline and specific } \\
\text { objectives }\end{array}$ \\
\hline 2. Questions & $\begin{array}{l}\text { 8. End users and settings: } 8 \mathrm{~b} \\
10 . \text { Health care questions: } 10 \mathrm{a}\end{array}$ \\
\hline $\begin{array}{l}\text { 5. Target population } \\
\text { preferences and views }\end{array}$ & $\begin{array}{l}\text { 14. Rationale/explanation for recom- } \\
\text { mendations: } 14 \mathrm{a}\end{array}$ \\
\hline $\begin{array}{l}\text { 9. Strengths and limitations of } \\
\text { the evidence }\end{array}$ & $\begin{array}{l}\text { 12. Assessment of the certainty of the } \\
\text { body of evidence }\end{array}$ \\
\hline $\begin{array}{l}\text { 10. Formulation of } \\
\text { recommendations }\end{array}$ & 15. Evidence to decision processes \\
\hline $\begin{array}{l}\text { 11. Consideration of benefits } \\
\text { and harms } \\
\text { 15. Specific and unambiguous } \\
\text { 16. Management options }\end{array}$ & 13. Recommendations: $13 \mathrm{a}, 13 \mathrm{~b}$ \\
\hline $\begin{array}{l}\text { 17. Identifiable key } \\
\text { recommendations }\end{array}$ & 2. Executive summary \\
\hline 20. Resource implications & $\begin{array}{l}\text { 14. Rationale/explanation for recom- } \\
\text { mendations: } 14 \mathrm{~b}\end{array}$ \\
\hline 23. Competing interests & $\begin{array}{l}\text { 19. Declaration and management of } \\
\text { interests: } 19 a \text { and } 19 b\end{array}$ \\
\hline
\end{tabular}

*AGREE includes six domains: domain 1, scope and purpose (items 13); domain 2, stakeholder involvement (items 4-6); domain 3, rigor of development (items 7-14); domain 4, clarity of presentation (items $15-$ 17); domain 5, applicability (items 18-21); and domain 6, editorial independence (items 22-23)

$\uparrow$ RIGHT includes seven sections/topics: topic 1, basic information (items la-c, 2-4); topic 2, background (items 5, 6, 7a, b,8a, b,9a, b); topic 3, evidence (items $10 a, b, 11 a, b, 12$ ); topic 4 , recommendations (items $13 a-c, 14 a-c, 15) ;$ topic 5 , review and quality assurance (items 16-17); topic 6, funding and declaration and management of interests (items $18 a, b, 19 a, b) ;$ and topic 7 , other information (items 20-22) 
Table 2 Differences Between the AGREE and RIGHT Reporting Checklists

\begin{tabular}{ll}
\hline AGREE (item) & RIGHT (item) \\
& Consistency and discrepancy between \\
AGRE and RIGHT
\end{tabular}

Items in AGREE that are partially stated in RIGHT

6. Target users 8. End users and settings: $8 \mathrm{a}$

7. Search methods 11. SRs $11 \mathrm{~b}$

13. External review 16. External review

Items in RIGHT that are partially stated in AGREE

3. Population

7. Target population: $7 \mathrm{a}$, $7 \mathrm{~b}$

4. Group membership

9. CPG development groups: $9 \mathrm{a}, 9 \mathrm{~b}$

22. Funding body

18. Funding sources and roles of the funder: $18 \mathrm{a}$, $18 \mathrm{~b}$

Items are intersected between AGREE and RIGHT 12. Link between recommendations and 13. Recommendations: evidence $13 \mathrm{c}$

19.Implementation

20. Access advice/tools

Items in AGREE only 8. Evidence selection criteria

NA

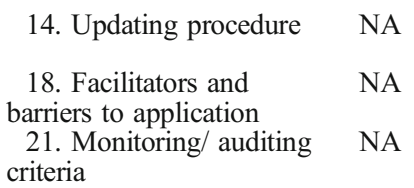

Items in RIGHT only

NA

1. Title/subtitle: $1 \mathrm{a}, 1 \mathrm{~b}, 1 \mathrm{c}$

NA
Both emphasize reporting the intended users of the CPG; AGREE additionally requires reporting "How the CPG may be used by its target audience (e.g., to inform clinical decisions, to inform policy, to inform standards of care)."

AGREE requires reporting details of the strategy used to search for evidence, which can include both strategy for searching existed SRs and primary literature search; RIGHT only emphasizes search strategy for existing SRs.

Both emphasize external review methods and how reviewers' comments are addressed to form recommendations; AGREE additionally requires "Purpose and intent of the external review (e.g., to gather feedback on the CPG draft, assess applicability and feasibility, disseminate evidence)."

Both emphasize that the target population should be described; RIGHT additionally requires one to "describe any subgroups that are given special consideration in the CPG." Both emphasize description of $\mathrm{CPG}$ developers' information; RIGHT additionally requires how the developers are selected.

Both emphasize the source of funding and that the funding body did not influence the CPG's content; RIGHT additionally requires the role of funding at the different stages of CPG development and in the dissemination and implementation.

Both emphasize the importance of evidence for recommendations; AGREE requires a link from key evidence and/or evidence summaries to each recommendation;

RIGHT emphasizes "the strength of recommendations and the certainty of the supporting evidence."

Both are related to CPG implementation; AGREE requires providing "advice and/or tools on how the recommendations can be applied in practice." RIGHT requires providing "where the CPG, its appendices, and other related documents can be accessed."

AGREE requires clarifying study selection criteria, such as target population characteristics; study design; outcomes; language (if relevant); context (if relevant). AGREE requires "describing the procedure for updating the CPG."

AGREE requires "describing the facilitators and barriers to the CPG's application." AGREE requires "providing monitoring and/or auditing criteria to measure the application of CPGs."

RIGHT requires "1a. Identify[ing] the report as a CPG. 1b. Describ[ing] the year of publication of the CPG. 1c. Describ[ing] the focus of the CPG, such as screening, diagnosis, or others."

RIGHT requires "defining new or key terms, and provid[ing] a list of abbreviations and acronyms if applicable."
Using RIGHT may leave it unclear as to how the target users should use the CPG.

Using RIGHT may be unclear if a primary literature search is needed and how it should be done.

Using RIGHT may not be transparent for the purpose of external review.

Using AGREE may be unclear if specific subgroups of population were considered.

Using AGREE may not be transparent on how CPG's developers were selected.

Using AGREE may be unclear on the influence of the funding body at different stages of CPG development.

Using AGREE may be unclear on how strong the recommendations are; using RIGHT may be unclear as to what evidence was linked to each recommendation.

Using RIGHT may be unclear on how the CPG will be implemented; using AGREE may be unclear on where these CPG relevant contents are.

Using RIGHT may be unclear on how the SR process was done.

Using RIGHT may be unclear on how the CPG will be maintained for currency.

Using RIGHT may be unclear on how the CPG will be applied.

Using RIGHT may unclear on how the CPG will be monitored or audited.

Using AGREE may be unclear on what components should be included in a title.

Using AGREE may be unclear if abbreviations and acronyms were provided. 
Table 2. (continued)

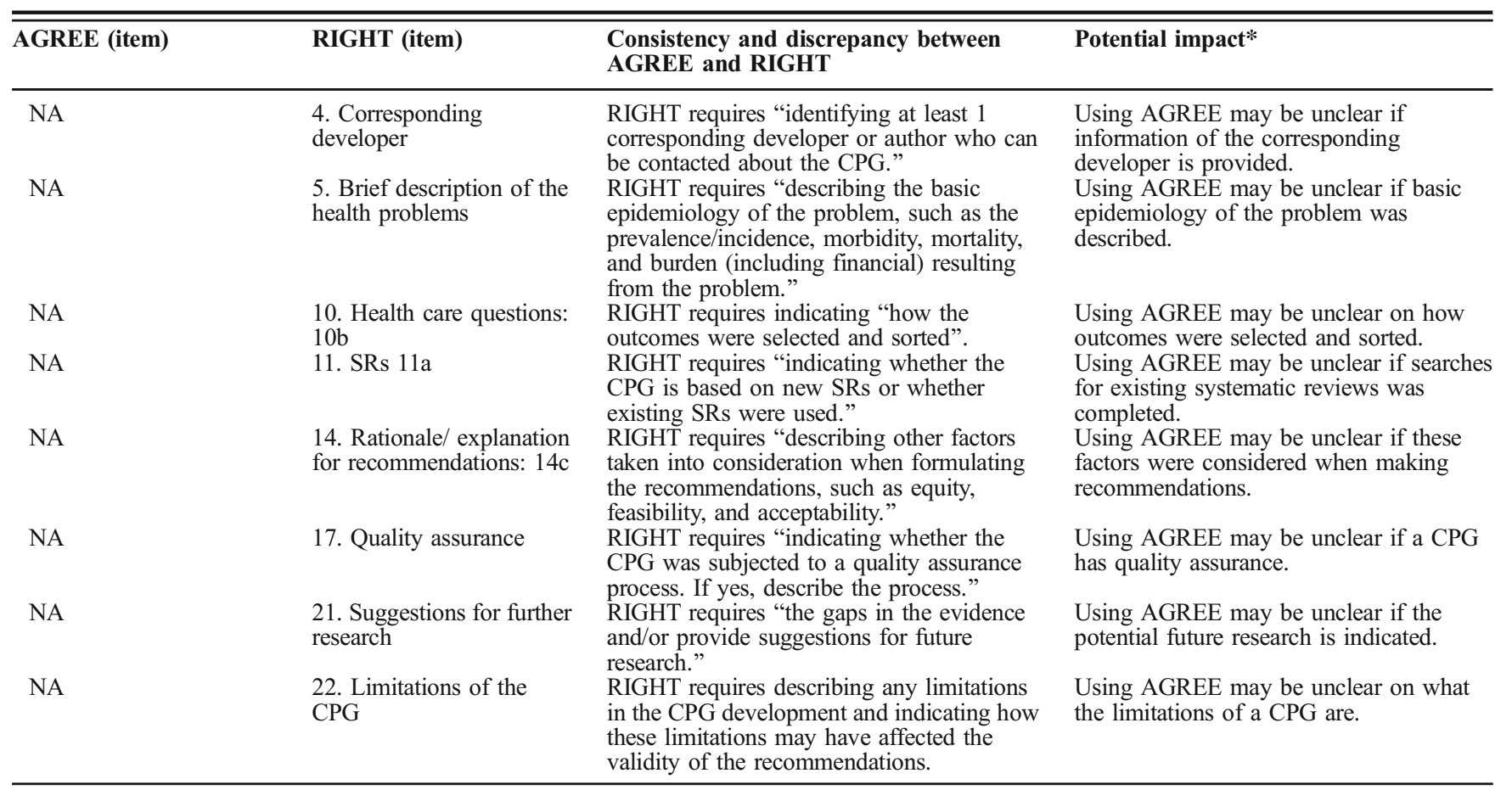

CPG clinical practice guideline, NA not available, SR systematic review

*The column only indicates the potential impact for the individual item. After other items in AGREE and RIGHT are executed, some potential impact listed in this column may be avoided

"Whether the CPG is based on new systematic reviews (SRs) or whether existing SRs were used" indicates that searching existing SRs is a necessary step when conducting a CPG. "Describing other factors taken into consideration when formulating the recommendations, such as equity, feasibility, and acceptability" can remind CPG developers to consider different factors when making recommendations. Noting "Whether the CPG was subjected to a quality assurance process," "The gaps in the evidence and/or provide suggestions for future research," and "Any limitations in the CPG development and indicating how these limitations may have affected the validity of the recommendations" are good reminders for $\mathrm{CPG}$ developers to keep the CPG transparent and consider ideas for future research.

Conversely, AGREE emphasizes "Evidence selection criteria" from a primary literature search; without this information, readers will not know how the SR was conducted. "Describing the procedure for updating the CPG" shows that any CPG should be updated over time and an out-of-date CPG may cause harm towards patients. "The facilitators and barriers to the CPG's application" and "Monitoring and/or auditing criteria to measure the application of CPGs" inform of the benefits, challenges, and requirements of CPG application.

It is worth noting that the AGREE reporting checklist 5 differs from the AGREE II assessment tool ${ }^{2}$ because the former is used to help CPG developers and authors determine how to report a CPG once it is complete, whereas the latter is an assessment tool used to appraise the quality of an existing CPG. As an assessment tool, AGREE II has a scoring system to calculate a quality score for each of the six domains, but the six domain scores are independent and should not be aggregated into a single quality score 7.

The AGREE or RIGHT reporting checklist, as other clinical research reporting checklists available on the EQUATOR Network ${ }^{4}$, only request the CPG developers or authors to list the items that are recommended. As a reporting checklist, it should not have a scoring system. Thus, we think that it is inappropriate to compare and report the correlation between the AGREE II assessment tool and a CPG reporting checklist (i.e., RIGHT) for one CPG (e.g., Wayant et al.'s paper ${ }^{8}$ ) since they were developed for different purposes.

We recommend that $\mathrm{CPG}$ developers use either AGREE plus items unique to RIGHT or RIGHT plus items unique to AGREE to ensure their CPG reporting is complete. We would suggest that in the near future, the authors of AGREE and RIGHT reporting checklists should collaborate to develop a new CPG reporting checklist to incorporate the strengths from both current checklists.

Corresponding Author: Ivan D. Florez, MD, MSc, PhD(c); Department of Pediatrics, School of MedicineUniversity of Antioquia, Calle 70 No. 52-21 050001, Medellín, Colombia (e-mail: ivan.florez@udea.edu. co). 
Authors' contribution XY and IF designed this study. XY, JM, and IF drafted the first manuscript. All the authors analyzed, interpreted data, and revised and approved the final manuscript version.

\section{Compliance with Ethical Standards:}

Conflict of Interest: There are no financial relationships with any organizations that might have an interest in the submitted work. XY and IF are users of AGREE reporting checklist and AGREE II assessment tool. IF is member of the AGREE collaboration. $Q W$ is one of the coauthors of the RIGHT reporting checklist publication. Other co-authors have declared no conflicts of interest.

\section{REFERENCES}

1. Graham R, Mancher M, Wolman DM, Greenfield S, Steinberg E, eds. Clinical Practice GuidelinesWe Can Trust. Washington, DC: National Academies Press; 2011, available at http://iom.nationalacademies.org/ reports/2011/. Accessed August 20, 2019.

2. Brouwers MC, Kho ME, Browman GP, et al. AGREE II: advancing guideline development, reporting and evaluation in health care. CMAJ. 2010;182(18):E839-42. https://doi.org/10.1503/cmaj.090449
3. Guyatt G, Oxman AD, Ak1 EA, et al. GRADE guidelines: 1. IntroductionGRADE evidence profiles and summary of findings tables. J Clin Epidemiol 2011;64(4):383-94. https://doi.org/10.1016/j.jclinepi.2010.04.026

4. EQUATOR network. Enhancing the QUAlity and Transparency of health Research. Reporting guidelines for main study types. http://www.equatornetwork.org/. Aaccessed August 20, 2019

5. Brouwers MC, Kerkvliet $\mathbf{K}$, Spithoff $\mathbf{K}$. The AGREE Reporting Checklist: a tool to improve reporting of clinical practice guidelines. BMJ. 2016;352:il 152. https://doi.org/10.1136/bmj.i1152

6. Chen Y, Yang $\mathbf{K}$, Marusic A, et al. A Reporting Tool for Practice Guidelines in Health Care: The RIGHT Statement. Ann Intern Med 2017;166(2):12832. https://doi.org/10.7326/m16-1565

7. https://www.agreetrust.org/. Aaccess August 20, 2019.

8. Wayant C, Cooper C, Turner D, Vassar M. Evaluation of the NCCN guidelines using the RIGHT Statement and AGREE-II instrument: a crosssectional review. BMJ Evid Based Med. 2019. https://doi.org/10.1136/ bmjebm-2018-111153

Publisher's Note Springer Nature remains neutral with regard to jurisdictional claims in published maps and institutional affiliations. 